\title{
Cardiovascular Safety and Superiority of Anti-Obesity Medications
}

\author{
Muath Alobaida (iD ${ }^{\text {I,* }}$ \\ Abdullah Alrumayh ${ }^{1, *}$ \\ Ayodipupo S Oguntade ${ }^{2}$ \\ Faez Al-Amodi ${ }^{3}$ \\ Mwango Bwalya ${ }^{3}$ \\ 'Department of Basic Sciences, Prince \\ Sultan bin Abdulaziz College for \\ Emergency Medical Services, King Saud \\ University, Riyadh, Kingdom of Saudi \\ Arabia; ${ }^{2}$ Nuffield Department of \\ Population Health, University of Oxford, \\ Oxford, UK; ${ }^{3}$ Institute of Cardiovascular \\ Science, University College London, \\ London, UK
}

*These authors contributed equally to this work
Correspondence: Muath Alobaida; Abdullah Alrumayh

Department of Basic Science, Prince Sultan bin Abdulaziz College for Emergency Medical Services, King Saud University, Riyadh, Kingdom of Saudi Arabia

Email malobaidal@ksu.edu.sa;

aalrumayh@ksu.edu.sa

\begin{abstract}
Over the past few decades, several anti-obesity medications have demonstrated an association with adverse cardiovascular outcomes, leading to their market withdrawal. This has caused researchers to investigate the cardiovascular safety of such medications in cardiovascular outcome trials. However, the data from these trials are limited, and their outcomes are not promising. Therefore, the aim of this review is to provide an overview of the current and past Food and Drug Administration-approved medications for weight loss, including novel diabetes medications (glucagon-like peptide 1 receptor agonists and sodiumglucose co-transporter-2 inhibitors) and non-diabetes medications, and to highlight the current designs of cardiovascular outcome trials and their importance in the evaluation of the overall safety concerns associated with these anti-obesity medications. The limitations of the trials and opportunities for improvement were also evaluated. Finally, we also briefly describe cardiovascular safety and risks in this review.
\end{abstract}

Keywords: anti-obesity medication, cardiovascular diseases, cardiovascular outcome trials, type 2 diabetes mellitus, obesity, obesity therapy

\section{Introduction}

Cardiovascular disease (CVD) is the primary cause of death worldwide, with approximately 18 million patient deaths in 2016, according to the World Health Organization (WHO). ${ }^{1}$ The prevalence of obesity has trebled over the last four decades. $^{2}$ Obesity is linked to a variety of chronic conditions, such as type 2 diabetes mellitus (T2DM), hypertension (HTN), stroke, and CV mortality. ${ }^{3}$ Consequently, for at-risk individuals, obesity may lead to the deterioration of health, poorer quality of life, and increased morbidity and mortality. ${ }^{4}$ It was noted that calorie overload leads to altered adipose tissue distribution, which is known to affect obesity-related CVD. ${ }^{5}$ Therefore, early recognition and intervention through counselling and medication are necessary for those who have obesity or increased adiposity and may help improve obesity-related morbidities. Currently, weight loss guidance suggests that, for some patients, adding pharmacotherapy to lifestyle adjustments can achieve long-term weight loss management. ${ }^{3,6}$ Further, since many anti-obesity medications have demonstrated an association with adverse CV outcomes leading to their market withdrawal, cardiovascular outcome trials (CVOTs) have been conducted to investigate their safety and superiority related to $\mathrm{CV}$ health in the long term. Unfortunately, these trials are still limited and there is a limited spectrum of medications for the management of obesity. The aim of this review is to provide an overview of the current and past Food and Drug 
Administration (FDA)-approved medications for weight loss and to discuss the importance of proper design of CVOTs in the evaluation of the overall safety concerns associated with these AOMs. We searched MEDLINE and EMBASE for articles on anti-obesity medication for cardiovascular safety and superiority published in the English language from 1997 to 2021 as part of larger trials and projects on anti-obesity medications. Keywords associated with "anti-obesity agents", "cardiovascular diseases", "type 2 diabetes mellitus", and "cardiovascular risk" were used for the search.

\section{Current Status of Anti-Obesity Medications}

There are several approaches to achieving weight loss. Although lifestyle modifications are the first line of treatment for obesity, several AOMs have been developed in the past few decades as a form of supplementation therapy. Pharmacotherapy and lifestyle modification have been endorsed for individuals who have a body mass index (BMI) $\geq 30 \mathrm{~kg} / \mathrm{m}^{2}$ or $\geq 27 \mathrm{~kg} / \mathrm{m}^{2}$ and at least one weightrelated comorbidity, such as T2DM, HTN, dyslipidaemia, or CVD. ${ }^{2}$ In addition, pharmacotherapy is an option for those who are not able to achieve clinically significant weight loss with lifestyle modification alone (regular exercise with a balanced diet). ${ }^{7}$

However, self-adjustment of lifestyle-related risk factors are mostly unsatisfactory, perhaps novel and safe weight loss medication is needed, possibly as an adjunct to other interventions. Just very recently, semaglutide is the first and only glucagon-like peptide-1 (GLP-1) receptor agonist therapy that has been granted approval for weight management in people who are overweight or obese. $^{8}$

\section{Assessment of the Cardiovascular Safety and Efficacy of Anti-Obesity Medications}

Historically, in the US and Europe, the pre-approval assessment of novel anti-obesity and anti-diabetic medications about $\mathrm{CV}$ safety and efficacy has not been clearly established. Over the past two decades, high exposure to $\mathrm{CV}$ risks and outcomes has led to post-approval withdrawals of AOMs (fenfluramine, ${ }^{9}$ sibutramine, ${ }^{10}$ and more recently, lorcaserin ${ }^{11}$ ) and diabetes medications (rosiglitazone $^{12}$ and muraglitazar ${ }^{13}$ ). These failures have highlighted the importance of developing new criteria for the pre-approval evaluation of these drugs and their effects on major adverse cardiovascular events (MACE) and, in some cases, post-approval surveillance procedures. ${ }^{14}$ However, some of these pre-approval and post-approval trials were not properly designed to assess or evaluate the excess risk of these AOMs. This indicates the importance of re-designing these trials to largely demonstrate the safety and efficacy of AOMs.

Although improvements in health markers (such as $\mathrm{CV}$ and cardiometabolic markers) are evident relatively quickly after weight loss, these benefits may not clearly outweigh the risk profile of an AOM. Conversely, benefits may take longer than expected to manifest; thus, early conclusions regarding unfavourable risk profiles can result in early misjudgment of the actual weight loss benefits of the medication. Furthermore, certain serious adverse events, such as cancer and CV risk factors, may take far longer to emerge; for example, lorcaserin, which had the serious adverse effect of possibly increasing the risk of cancer, was withdrawn a few years after market approval. Thus, the time to improvement and adverse events in an enriched population with complex comorbidities (eg, diabetes, CVD, and obesity sleep apnoea) should be considered in the assessment of AOMs. CVOTs of novel AOMs with longer-term safety data are necessary for a more accurate depiction of AOMs.

\section{The Design of Cardiovascular Outcome Trials}

Despite the previous CV safety concerns regarding AOMs, fewer CVOTs have been conducted, especially when compared with trials on antidiabetic medications (such as, GLP1 RA and SGLT2i). According to regulation guidelines (US-FDA and European Medicines Agency [EMA]), all novel AOMs must induce weight loss of 5\% or higher after 12 months of treatment; this is the benchmark for all CVOTs. Although there is some variability to this rule, it exists to prevent the activation of "stopping" rules by the regulatory authorities. ${ }^{15}$

Currently, novel AOMs may be subjected to postauthorization based on the hazard ratio (HR) of their randomised controlled trials (RCTs). However, these optional criteria have contributed to several withdrawals of AOMs and underpin the need for post-approval safety studies in real-life conditions. Limitation of RCTs including, applicability to a very narrow population, often costly, might take longer to complete and the insufficient number 
of patient recruitment into RCTs due to rare diseases. For these reasons, some suggested real-world database (RWD) as alternatives to RCTs. ${ }^{16}$ The use of real-world data, such as electronic health records and administrative claims, is being considered for assessing the safety and effectiveness of newly approved products and satisfy the post-approval requirement. ${ }^{17,18}$ It can assess the excluded population from RCT (eg, pregnant women, older population, comedication patterns and people with many comorbidities) with less cost and in less time than RCTs.

Specific research questions, regulatory use, and data characteristics are vital for a meaningful conclusion that determines whether the real-world database can be a fit for regulatory purposes (ie, FDA approval). ${ }^{18}$ In fact, framing the clinical question determines whether RWD can substitute for an RCT of interest. ${ }^{16}$ Moreover, to produce meaningful interpretable results, it is necessary to have valid, relevant research definitions for disease, outcomes, and treatments. It is needed to have a framework for assessing the utility, usefulness and representativeness of a population of interest of the real-world data in relation to a specific research question and the intended regulatory purpose. However, given the clinical research question and intended regulatory use, more guidance is required on the basis of what evidence of validation for critical data elements is acceptable.

When comparing both approaches, on average realworld data and randomised controlled trials result in similar findings. ${ }^{19-21}$

There are many examples where analyses of RWD yield a successful confirmation later by randomised controlled trials. For example, RWD analysis of in-hospital CABG patients showed an elevated risk of mortality among those receiving aprotinin compared with aminocaproic acid. ${ }^{22}$ Later, the BART trial confirmed the elevated risk of the RWD analysis that subsequently resulted in the US market withdrawal of aprotinin. ${ }^{23}$ Similarly, a large US commercial health database has shown the almost similar positive result of canagliflozin on the risk of $\mathrm{HF}$ hospitalisations. ${ }^{24,25}$ Therefore, it is plausible that understanding why some real-world studies fail while others succeed can build confidence and increase greater reliance on real-world studies for regulatory decision approval. Even though RCTs are the gold standard for evaluating efficacy, the generalizability of their safety data to clinical practice can be limited due to their strict inclusion and exclusion criteria. ${ }^{26}$
In relation to endpoints that being used in the $\mathrm{CV}$ outcome trials, some previous trials that used 3-point (3P)MACE and 3P-MACE-plus (Table 1) as primary and secondary outcomes, respectively, have added valuable insights beyond the issue of safety. For example, MACEs permit simultaneous performance of trials and analysis of vital clinical outcomes in CVOTs. In addition, they capture $\mathrm{CV}$ outcomes that are clinically relevant, identify the potential treatment effects of AOMs, and can be implemented easily; moreover, the 3P-MACEs provide better statistical efficiency. Thus, regulatory authorities mandate the use of 3P-MACEs as a primary composite endpoint in large CVOTs of AOMs and anti-diabetic medications.

The recommended guidelines (EMA and US-FDA) demonstrate the requirements for CVOTs (Table 1)

Table I Key Points for Cardiovascular Outcome Trials According to the European Medicine Agency Guidelines for Assessing Cardiovascular Risk

\begin{tabular}{|l|l|}
\hline \multicolumn{2}{|c|}{ Requirements for Cardiovascular Outcome Trials } \\
\hline Population & $\begin{array}{l}\text { The recruited population needs to be at } \\
\text { a higher risk of having CV events, ie, MACE }\end{array}$ \\
\hline Safety outcomes & $\begin{array}{l}\text { Primary composite MACE: CV-related } \\
\text { death, non-fatal myocardial infarction, and } \\
\text { non-fatal stroke are the preferred safety } \\
\text { outcomes. } \\
\text { Can also include 3P-MACE-plus: } \\
\text { hospitalisation for unstable angina, heart } \\
\text { failure, and possibly other endpoints. } \\
\text { Sufficient number of MACEs and sufficient } \\
\text { power illuminate non-excessive risks }\end{array}$ \\
\hline $\begin{array}{l}\text { Quantification of } \\
\text { cardiovascular risk }\end{array}$ & $\begin{array}{l}\text { The analysis of cardiovascular events may } \\
\text { include meta-analysis or large, separate, CV- } \\
\text { safety trial of the new agent demonstrating } \\
\text { an HR upper limit (95\% Cl, two-sided) <I.8. } \\
\text { If the HR falls between I.3 -I.8, post- } \\
\text { marketing trial may be required to reaffirm } \\
\text { and show that the HR is <I.3. } \\
\text { Superiority, which means that the HR was } \\
<\text { LI.3 in the pre-marketing trial, indicates } \\
\text { that no further post-marketing trial is } \\
\text { required. }\end{array}$ \\
\hline Evaluation of results & $\begin{array}{l}\text { Illustrating the mechanism of action and } \\
\text { effects on known CV risk factors. } \\
\text { Additional CVOTs may be requested if } \\
\text { there are any indications of high risk of CV } \\
\text { events or a lack of precision. }\end{array}$ \\
\hline
\end{tabular}

Abbreviations: $\mathrm{Cl}$, confidence interval; $\mathrm{CV}$, cardiovascular; $\mathrm{HR}$, hazard ratio; MACE, major adverse cardiovascular events. 
regarding how to answer safety and efficacy questions, how to show an adequate number of events in a specific time ( $>600$ primary endpoint events to rule out an $\mathrm{HR} \geq$ 1.3 for 3P-MACE), and how to determine if a subject is at a high risk of having $\mathrm{CV}$ events, in order to show the defined outcome (ie, CV mortality or events). Patientspecific characterisation creates a lack of population generalizability, in addition to delays in regulatory advisory approval.

There are some limitations to the current CVOTs. They include lack of generalizability and shorter timeline to potential harm and benefits. Notably, including people with predominantly very high or very low risk of having $\mathrm{CV}$ events or mortality in a trial may limit its generalizability in the real world. In addition, although 5 years of $\mathrm{CV}$ assessment for potential benefits is reasonable, $\mathrm{CV}$ benefits with a long latency period may not be apparent during the 5-year period of treatment. Similarly, the likelihood of detecting CV risk in short-term CVOTs $(<3$ years) is low since the MACE outcomes are not assessed after 5 years of randomisation treatment. Thus, the CVOTs are event-driven, not time-driven. On the other hand, biases and problem, such as loss of follow-up are common in RCTs. A systematic review of 117 trials found almost $80 \%$ of them showed a loss of follow-ups, and approximately one in six CVOTs may have a change in the primary outcome. ${ }^{27}$ Thus, prevention must be prioritised; and authors should report and provide the baseline characteristics of those participants, the extent of follow-up prior exclusion, and time of dropout and address that implication of those participants when interpreting results. More importantly, Inadequate allocation concealment may drive differential treatment effects (either overestimation or underestimation ${ }^{28}$ ) and considered as an independent factor associated with loss of follow-ups, as allocation concealment was inadequate or unclear in more than $50 \%$ of the trials.

Considering these limitations, researchers should consider using diverse low-risk populations, longer follow-up timelines, and maintenance of standardised definitions when designing future trials. Firstly, expanding the population to include low-risk participants whose CVD has not yet been established would add valuable information to the assessment of the use of AOMs in these populations. Secondly, designing trials with a longer follow-up timeline (albeit a lifelong follow-up) helps identify safety issues, ensure that benefits are not missed (in case of short-term trials), and provides credible cost-effectiveness data; in addition, longer follow-up could improve drug adherence and continuity over time. Lastly, a standardised definition of strong $\mathrm{CV}$ outcomes would produce an excellent and fair comparison to the other new agents in terms of $\mathrm{CV}$ safety and efficacy. These major CV outcomes, including CV-related death, non-fatal myocardial infarction, and non-fatal stroke, are combined in 3P-MACE, which can be further expanded into 3P-MACE plus to include hospitalisation for heart failure or unstable angina. These have demonstrated strong $\mathrm{CV}$ outcomes to show the CV safety and efficacy of an AOM.

It is important to note that the enrolled population must be more compliant with treatment in clinical trials and must have a low discontinuation rate. The following sections will review AOMs, focusing on their efficacy and safety in patients with CVD, as summarised in Table 2.

\section{Anorexigens}

\section{Phentermine/Topiramate}

Phentermine/topiramate is a combination of a sympathomimetic agent (phentermine), which functions as an appetite suppressant, and an antiepileptic (topiramate) agent, which acts as a potent inhibitor of carbonic anhydrase enzymes and is thought to reduce appetite by altering food taste. However, the definitive mechanism behind the weight reduction benefits of the medication is not explicit. This drug combination leads to weight loss and an increase in $\mathrm{CV}$ risk factors. Although phentermine is a sympathomimetic agent that potentially leads to increased heart rate and blood pressure, ${ }^{29}$ the drug may show an exception with a minimal sympathomimetic reaction due to the weight loss effect. ${ }^{30}$ The use of phentermine/topiramate has been evaluated in two trials: the CONQUER trials and a two-year-extension SEQUEL trial. $^{31}$ The SEQUEL trial showed enhanced cardiometabolic outcomes and continued weight loss of $-9.3 \%$, $-10.5 \%$, and $-1.8 \%$ for two different doses of phentermine/topiramate $(7.5 \mathrm{mg} / 45 \mathrm{mg}$ and $15 \mathrm{mg} / 92 \mathrm{mg})$ compared with the placebo $(\mathrm{P}<0.0001)$. Similarly, CONQUER showed continued weight losses of $-8.1 \mathrm{~kg},-10.2 \mathrm{~kg}$, and $-1.4 \mathrm{~kg}$ for the two different doses of the medication (7.5mg/45mg and $15 \mathrm{mg} / 92 \mathrm{mg}$ ) compared with the placebo $(\mathrm{P}<0.0001)$. Even though there were no major differences between the rates of MACE in the two groups, ${ }^{29}$ more extensive trials with higher statistical power need to be conducted to evaluate the CV safety of the drug with greater certainty. Moreover, the EMA banned the drug, 


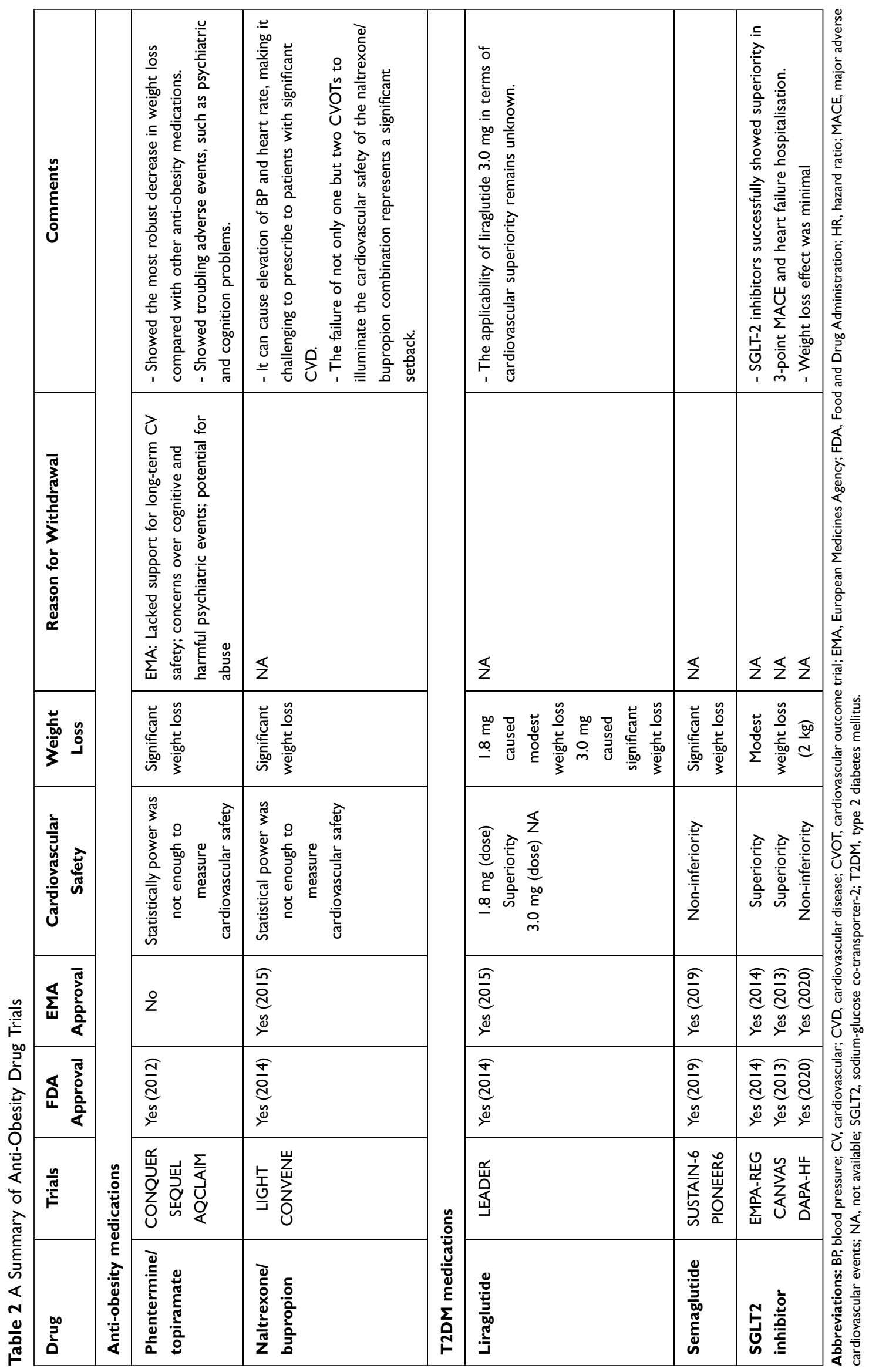


claiming that it lacked support for long-term CV safety, and raised fears over cognitive events (eg, problems with memory and attention) and psychiatric effects (eg, depression and anxiety). ${ }^{32}$ Nonetheless, the AQCLAIM CVOT is currently being conducted to study the safety of phentermine plus topiramate on the CV system. In conclusion, phentermine/topiramate has shown the most robust decrease in weight-loss compared with other AOMs. Notably, increased adverse effects, such as neuropsychiatric events, indicate that strict control of the drug is required. Phentermine/topiramate is not the only AOM that was withdrawn from the market in relation to safety issues. Some AOM medications, such as lorcaserin, which were associated with similar concerns, were withdrawn as well; lorcaserin was withdrawn from the market as it was associated with the development of cancer. This underpins the need for AOMs to meet safety requirements.

\section{Naltrexone/Bupropion}

Naltrexone is an opioid receptor antagonist, whereas bupropion is a norepinephrine-dopamine reuptake inhibitor and nicotinic receptor antagonist. This drug combination was designed to reduce hunger without any impact on energy expenditure. However, its mechanism of action is not completely clear. Furthermore, like other anti-obesity agents (phentermine and sibutramine), bupropion has sympathomimetic effects; thus, there is increased concern over its effect on CV safety, such as increased heart rate and blood pressure. ${ }^{33}$ Both the FDA and EMA approved the drug for the market in 2014 and 2015, respectively. Trials, such as the LIGHT and CONVENE trials, have been conducted to evaluate the long-term safety of the drug in terms of the CV system. However, the LIGHT trial was terminated in 2015 after an early release of interim data, which showed a risk of bias. ${ }^{34}$ Despite its termination, interpretation of the $50 \%$ interim result showed no significant reduction of MACE with naltrexone/bupropion (HR did not exceed 2.0). The CONVENE trial was stopped prematurely due to its short trial period and lack of adequate patient follow-up. The lack of sufficient data did not allow for a statistical exploration. ${ }^{35}$

\section{Anti-Diabetic Medications with Weight Loss Benefits}

Obesity and T2DM are interrelated and strongly associated with an increased risk of CVD. ${ }^{36}$ Since some AOMs (eg, phentermine/topiramate-ER and lorcaserin) improve glycaemic control in patients with diabetes, new glucose-lowering drugs (ie, glucagon-like peptide-1 receptor agonists [GLP1RAs]) have also shown weight reduction benefits. Glucagonlike peptide-1 (GLP-1) is a gastrointestinal hormone secreted primarily from gut enteroendocrine cells in the distal colon and ileum in response to nutrient stimuli. GLP1-RAs stimulate insulin secretion, inhibit glucagon production, and slow gastric emptying, thereby regulating glycaemic control and inducing postprandial satiety and, eventually, weight loss. ${ }^{37-39}$ Several CVOTs of different GLP1-RAs, which were conducted using patients with T2DM and CVDs, demonstrated the beneficial glucose-lowering effects of the drugs in the treatment of T2DM. Compared to various GLP1-RAs, only one GLP1RA, liraglutide, has shown great benefits for weight loss in addition to its essential glucose-lowering effect and has received FDA approval as an AOM (Table 2).

\section{Liraglutide}

The FDA and EMA approved liraglutide, with the trade names Victoza (1.8 mg QD) and Saxenda (3.0 mg QD), for the management of $\mathrm{T} 2 \mathrm{DM}$ and obesity, respectively. ${ }^{40}$ A recent study showed that liraglutide has weight loss benefits (weight loss ranging from $4 \mathrm{~kg}$ to $6 \mathrm{~kg}$ ) as an adjunct to lifestyle and diet interventions in obese or overweight adolescents and adults who have at least one obesity-related comorbidity. The study was an RCT conducted using liraglutide $3.0 \mathrm{mg}$ for weight management (SCALE obesity and prediabetes); ${ }^{41} 3731$ obese or overweight adults who did not have T2DM but had a BMI of at least 30 or $27 \mathrm{~kg} / \mathrm{m}^{2}$ and either dyslipidaemia or HTN were included in the study. The liraglutide group (3.0 mg injection) showed a mean weight loss of $-8.4 \mathrm{~kg}$ vs $-2.8 \mathrm{~kg}$ for the placebo group. The proportion of patients who achieved at least $5 \%$ weight loss was $63.2 \%$ in the liraglutide group and $27.1 \%$ in the placebo group $(\mathrm{P}<0.001) ; 33.1 \%$ of those in the liraglutide group and $10.6 \%$ in the placebo group lost at least $10 \%$ of their weight $(\mathrm{P}<0.001)$. Liraglutide was associated with improvements in cardiometabolic risk factors. In another $\mathrm{RCT}^{42}$ liraglutide $3.0 \mathrm{mg}$ was used for weight management in 251 adolescents with obesity who did not respond to lifestyle intervention. Compared to the placebo plus lifestyle, the liraglutide group had a significantly greater reduction in the primary endpoint (BMI standard deviation score). Based on the results of these trials, liraglutide was approved for weight management. However, GLP1-RAs, specifically liraglutide, have received more attention after the first long-term CVOT (LEADER trial) of patients with T2DM and at a high risk of having CV events was 
conducted. After a median follow-up duration of 3.8 years, liraglutide $(1.8 \mathrm{mg} /$ day $)$ showed superiority over the placebo in the primary composite outcome (eg, time of CV death, non-fatal MI, and non-fatal stroke) (13\% vs $14.9 \%$, $\mathrm{p}=0.01$ for superiority). Compared with the placebo, patients on liraglutide also exhibited a lower risk of $\mathrm{CV}$ mortality, all-cause mortality, and microvascular events. More importantly, the liraglutide group also showed a favourable reduction in body weight $(-2.3 \mathrm{~kg}[95 \% \mathrm{CI}$, 2.5 to 2.0$]$ ) and blood pressure (systolic blood pressure: $-1.2 \mathrm{mmHg}[95 \% \mathrm{CI}, 1.9$ to 0.5$]$ and diastolic blood pressure: $-0.6 \mathrm{mmHg}$ [ $95 \% \mathrm{CI}, 0.2$ to 1.0$]$ ) compared with the placebo group. ${ }^{43}$ Due to the similarities in exposure to the liraglutide dose $(1.8 \mathrm{mg})$, the EMA found the long-term LEADER data to be relevant to the CV safety of liraglutide $3.0 \mathrm{mg}$ and approved it in June 2017 (even though liraglutide $1.8 \mathrm{mg}$ only was used in the LEADER trial). Thus, the applicability of liraglutide $3.0 \mathrm{mg}$ remains unknown and warrants further research. ${ }^{43}$ Finally, a posthoc analysis ${ }^{44}$ from the SCALE RCTs demonstrated that there is no association between liraglutide $(3.0 \mathrm{mg})$ and excessive CV risk. However, the study had limitations of statistical analysis, such as the wide confidence intervals of the effect size and the retrospective adjudication of events. Overall, given its beneficial effect on weight loss and glycaemic control, liraglutide could be the treatment of choice for patients with T2DM and established CVD (Table 2).

\section{Semaglutide}

Semaglutide, a GLP1-RA, was initially approved in 2017 as a once-weekly injection dose for the treatment of T2DM, and very recently, in 2021, it was approved for weight loss management. ${ }^{8}$ Initially, it was shown to cause greater weight loss than a placebo and liraglutide, as demonstrated in a phase-two, randomised, double-blind, dose-ranging trial. ${ }^{45}$ The trial compared the efficacy of semaglutide with those of liraglutide and a placebo in patients with obesity. In the trial, the mean weight loss of patients treated with semaglutide $(0.2 \mathrm{mg}$ and higher $)$ was significant: $11.2 \%$ to $13.8 \%$, compared with $7.8 \%$ for liraglutide and $2.3 \%$ in a placebo. ${ }^{45}$ Moreover, in SUSTAIN-6, a long-term CVOT, 3297 patients with T2DM who had been at high risk of having CV events for 2 years were treated with semaglutide (once-weekly). The result demonstrated that semaglutide achieved a significant reduction (6.6\%) in 3-P MACE (time to CV death, non-fatal MI, and non-fatal stroke) compared with the placebo (8.9\%) $(\mathrm{P}<0.001$ for non-inferiority). There was no difference between the groups in terms of mortality rates for heart failure hospitalisation, whether from $\mathrm{CV}$ or all-cause mortality. ${ }^{46}$ The weight loss in the $0.5 \mathrm{mg}$ and $1.0 \mathrm{mg}$ semaglutide groups was $2.9 \mathrm{~kg}$ and $4.3 \mathrm{~kg}$, respectively, compared to the placebo group $(\mathrm{P}<0.001$ for both comparisons). Significantly, compared with the LEADER trial, which was lengthier and broader in scope, this trial was designed to demonstrate safety instead of superiority. Interestingly, this trial achieved similar outcomes with fewer patients and fewer follow-up years. ${ }^{47}$ Subsequently, in 2019, PIONEER6, a CVOT, evaluated the effects of oral semaglutide in 3183 patients with T2DM and at high risk of having CV events. ${ }^{48}$ This trial confirmed the non-inferiority of oral semaglutide in 3-P MACE (time to CV death, non-fatal MI, and nonfatal stroke), CV mortality, and all-cause mortality compared with the placebo ( $\mathrm{P}<0.001$ for non-inferiority). The mean change in body weight with oral semaglutide was $-4.2 \mathrm{~kg}$, compared to the placebo value of $-0.8 \mathrm{~kg}$. Very recently, once-weekly injection of semaglutide $2.4 \mathrm{mg}$ has just been approved for weight management in obesity based on STEP (Semaglutide Treatment Effect in People with obesity) trials that consist of four clinical trials of semaglutide $2.4 \mathrm{mg}$ (once-weekly subcutaneous) in obesity, and has enrolled approximately 4500 adults with overweight or obesity (Table 2). ${ }^{8,49-52}$

In the STEP 1 trial, ${ }^{49} 1961$ patients with a BMI of 30 or higher (or $\geq 27$ with at least one coexisting comorbidities) were randomised to receive a once-weekly subcutaneous semaglutide $(2.4 \mathrm{mg})$ or placebo for 68 weeks with lifestyle intervention in both. The mean change in body weight was $-14.9 \%$ with semaglutide and $-2.4 \%$ with placebo, a difference of -12.4 percentage points. The results are encouraging, with significantly more patients in the semaglutide group having clinically significant weight loss. Also, there were decreases in cardiometabolic risk factors, as well as improvements in physical function and QoL. On the other hand, gastrointestinal (GI) were the most frequently reported adverse events that were mild to moderate in severity; however, it causes $7 \%$ discontinuation of semaglutide group compared with the placebo $3.1 \%$. The additional question could be raised about the efficacy of subcutaneous semaglutide in people with obesity and normal glucose tolerance since almost $75 \%$ of the study participants were white and males were underrepresented; in addition, $43 \%$ of the participants had prediabetes. $^{53}$ Thus, semaglutide $2.4 \mathrm{mg}$ once weekly 
plus lifestyle intervention was associated with a sustained, clinically relevant reduction in body weight for people with overweight or obesity.

The STEP 2 superiority trial ${ }^{50}$ assessed the efficacy and safety of once-weekly subcutaneous semaglutide $2.4 \mathrm{mg}$ vs semaglutide $1.0 \mathrm{mg}$ (the dose approved for diabetes treatment) and placebo for weight management in people with overweight or obesity, and type 2 diabetes. A total of 1210 participants were randomised to receive a once-weekly subcutaneous semaglutide $(2.4 \mathrm{mg})$, semaglutide $(1.0 \mathrm{mg})$ or placebo. The mean change in body weight was $-9.6 \%$ with semaglutide $2.4 \mathrm{mg}$ vs $-3.4 \%$ with placebo, a difference of $-6.2 \%$ (95\% CI -7.3 to -5.2 ; $\mathrm{p}<0.0001)$. Consistently, GI were most often reported with semaglutide $2.4 \mathrm{mg}(63.5 \%)$ and $1.0 \mathrm{mg}$ (57.5\%) than placebo $(34.3 \%)$. Thus, semaglutide $2.4 \mathrm{mg}$ has a superior decrease in body weight than the placebo and a clinically meaningful decrease in people with overweight or obesity and T2DM. More importantly, this trial was greater than that seen with liraglutide and other approved AOMs in similar populations.

The STEP 3 multinational randomised control trial ${ }^{51}$ assessed the efficacy of once-weekly subcutaneous semaglutide $2.4 \mathrm{mg}$ vs the placebo for weight management in adults with overweight or obesity as an adjunct to intensive behavioural therapy with an initial low-calorie diet for 68 weeks' treatment. A total of 611 participants with overweight or obesity, resulted in $16.0 \%$ reductions in body weight of semaglutide compared with $5.7 \%$ with the placebo; the difference was statistically significant $(-10.3$ percentage points [95\% CI, -12.0 to -8.6$]$; $\mathrm{P}<0.001$ ). Similarly, discontinuation of treatment due to these GI adverse events in semaglutide was $3.4 \%$ vs $0 \%$ of placebo participants.

Lastly, the STEP 4 trial $^{52}$ assessed the efficacy of continuing vs withdrawing of once-weekly subcutaneous semaglutide $2.4 \mathrm{mg}$ treatment on weight loss maintenance in people with overweight or obesity after a 20 -week run-in period. Following the 20 -week run-in period, those who continued on semaglutide $2.4 \mathrm{mg}$ from 20 to 68 weeks continued to lose weight with a mean change of $-7.9 \%$ vs $+6.9 \%$ with those who switched to the placebo (difference, $-14.8 \%$ [95\% CI, -16.0 to -13.5$]$; $\mathrm{P}<0.001$ ). Also, similarly to STEP 1 trial, there were decreases in cardiometabolic risk factors (ie, waist circumference, systolic blood pressure; $-9.7 \mathrm{~cm}[95 \% \mathrm{CI},-10.9$ to $-8.5 \mathrm{~cm}],-3.9 \mathrm{~mm} \mathrm{Hg}[95 \%$ CI, -5.8 to $-2.0 \mathrm{~mm} \mathrm{Hg}$ ]; all $\mathrm{P}<0.001$, respectively,), as well as improvements in physical function. Almost $80 \%$ of the participants are females that raise a limitation of generalisability among other gender and ethnicity.

Nevertheless, although there are multiple CVOTs on GLP1-RAs that are worthy of discussion, only semaglutide and liraglutide have been proven to have weight loss benefits with a cardiovascular safety risk, but further studies on semaglutide for MACE outcomes are needed. Whether they will benefit non-diabetic obese individuals remains unknown.

\section{Sodium-Glucose Co-Transporter-2 Inhibitors}

Sodium-glucose co-transporter-2 (SGLT2) inhibitors have been available on the market since 2012. SGLT2 is found in the proximal convoluted tubule and causes avid reabsorption of filtered glucose in the kidney. ${ }^{54}$ By inhibiting the glucosereabsorption process, glucosuria is increased, thereby decreasing blood glucose and preventing hyperglycaemia in patients with T2DM. As patients with diabetes are at risk of having CV events and renal diseases, SGLT2 inhibitors, specifically empagliflozin, canagliflozin, and dapagliflozin, demonstrated a significant reduction in 3-P MACE and heart failure hospitalisation in their CVOT trials [EMPA-REG: HR $-0.86,95 \%$ CI -0.74 to $0.99 ; \mathrm{p}<0.001$ for non-inferiority; $\mathrm{p}=0.04$ for superiority), (CANVAS: HR $-0.86,95 \% \mathrm{CI}-0.75$ to 0.97 ; $\mathrm{p}<$ 0.001 for non-inferiority; $\mathrm{p}=0.02$ for superiority), and (DAPAHF: HR $-0.74,95 \%$ CI -0.65 to $0.85 ; \mathrm{p}<0.001$ for noninferiority)]; however, the weight loss caused by these agents was modest ${ }^{55-57}$ (Table 2). In the three-year EMPA-REG trial, weight loss caused by these agents was only $2 \mathrm{~kg}$. However, a modest reduction of body weight was associated with dapagliflozin plus placebo compared with exenatide (GLP1-RA) plus placebo $(-2.28$ vs -1.51$)$, as demonstrated in the DURATION-8 trial. ${ }^{58}$ Very recently, VERTIS CV, a CVOT, demonstrated a mean weight loss of $2.4 \mathrm{~kg}$ for ertugliflozin $5 \mathrm{mg}$ vs placebo and $2.8 \mathrm{~kg}$ for ertugliflozin $15 \mathrm{mg}$ vs placebo. ${ }^{59}$ Nevertheless, GLP1-RAs offer more profound body weight reduction compared with SGLT2i; however, both are great in preventing weight gain compared with other classes of glucose-lowering agents. Despite all the associated benefits of these agents, it is essential to keep in mind that lifestyle interventions (ie, diet and exercise) are vital for reaping their maximum benefits.

\section{Conclusion}

In summary, obesity is a well-known risk factor for CVD. Clinical trials of AOMs as a weight loss intervention have yielded controversial results, and agencies such as the FDA 
and EMA have introduced recommendations for improving the CV safety of these medications. This review concludes that AOMs should deliver safe long-term CV outcomes and have favourable superiority. Currently, only a few drugs have managed to achieve adequate cardiovascular safety and superiority. This underpins the need to adjust and introduce new designs for future CVOTs. Presently, the future of AOMs is uncertain, as regulatory authorities and physicians do not view obesity medication as a global priority. This further increases the challenge of comparing the $\mathrm{CV}$ benefits of new AOMs to those of other established medications, such as antihypertensives or statins.

\section{Funding}

This work was self-funded by the authors.

\section{Disclosure}

The authors declare that they have no competing interests.

\section{References}

1. World Health Organization. Cardiovascular Diseases (CVDs) Fact Sheets. World Health Organization; 2016.

2. World Health Organization. Fact Sheet: Obesity and Overweight. World Health Organization; 2020.

3. Jensen MD, Ryan DH, Apovian CM, et al. 2013 AHA/ACC/TOS guideline for obesity in adults: a report of the American College of Cardiology/American Heart Association task force on society. $J \mathrm{Am}$ Coll Cardiol. 2014;63(25 Pt $\quad$ B):2985-3023. doi:10.1016/j. jacc.2013.11.004

4. Sharma AM, Kushner RF. A proposed clinical staging system for obesity. Int J Obes. 2009;33(3):289-295. doi:10.1038/ijo.2009.2

5. Benotti PN, Wood GC, Carey DJ, et al. Gastric bypass surgery produces a durable reduction in cardiovascular disease risk factors and reduces the long-term risks of congestive heart failure. $J \mathrm{Am}$ Heart Assoc. 2017;6(5). doi:10.1161/JAHA.116.005126

6. Garvey W, Garber A, Mechanick J, et al. American association of clinical endocrinologists and American College of Endocrinology position statement on the 2014 advanced framework for a new diagnosis of obesity as a chronic disease. Endocr Pract. 2014;20 (9):977-989. doi:10.4158/EP14280.PS

7. Kim GW, Lin JE, Blomain ES, Waldman SA. New advances in models and strategies for developing anti-obesity drugs. Expert Opin Drug Discov. 2013;8(6):655-671. doi:10.1517/ 17460441.2013.792804

8. FDA. FDA approves new drug treatment for chronic weight management, first since 2014 [Internet]. FDA Drug Safety Communication; 2021. Available from: https://www.fda.gov/news-events/pressannouncements/fda-approves-new-drug-treatment-chronic-weightmanagement-first-2014. Accessed June 29, 2021.

9. Connolly HM, Crary JL, McGoon MD, et al. Valvular heart disease associated with fenfluramine-phentermine. N Engl J Med. 1997;337 (24):1772-1776. Available from: http://www.nejm.org/doi/abs/10. 1056/NEJM199712113372414.

10. James WPT, Caterson ID, Coutinho W, et al. Effect of sibutramine on cardiovascular outcomes in overweight and obese subjects. $N$ Engl $J$ Med. 2010;363(10):905-917. doi:10.1056/NEJMoa1003114
11. FDA. Belviq, Belviq XR (lorcaserin) by Eisai: drug safety communication - FDA requests withdrawal of weight-loss drug. U.S. Food and Drug Administration; 2020.

12. U.S. Food and Drug Administration. FDA Requires Removal of Some Prescribing and Dispensing Restrictions for Rosiglitazone-Containing Diabetes Medicines. FDA Drug Safety Communication; 2013.

13. Nissen SE, Wolski K, Topol EJ. Effect of muraglitazar on death and major adverse cardiovascular events in patients with type 2 diabetes mellitus. J Am Med Assoc. 2005;294(20):2581. doi:10.1001/jama.294.20.joc50147

14. Lopaschuk G, Kuzo RS, Gerber TC. Effect of rosiglitazone on the risk of myocardial infarction and death from cardiovascular causes: commentary. Heart Metab. 2007;34:5-9.

15. Patel DK, Stanford FC. Safety and tolerability of new-generation anti-obesity medications: a narrative review. Postgrad Med. 2018;130(2):173-182. doi:10.1080/00325481.2018.1435129

16. Franklin JM, Schneeweiss S. When and how can real world data analyses substitute for randomized controlled trials? Clin Pharmacol Ther. 2017;102(6):924-933.:

17. Framework for FDA's real-world evidence program [Internet]; 2018 [cited 2021 Jun 8]. Available from: www.fda.gov.

18. Bryan J. Characterizing RWD Quality and Relevancy for Regulatory Purposes. 2018.

19. Anglemyer A, Horvath HT, Bero L. Healthcare outcomes assessed with observational study designs compared with those assessed in randomized trials. Cochrane Database Syst Rev. 2014;2014(4). doi: 10.1002/14651858.MR000034.pub2

20. Concato J, Shah N, Horwitz RI. Randomized, controlled trials, observational studies, and the hierarchy of research designs. $N$ Engl J Med. 2000;342(25):1887-1892. doi:10.1056/NEJM200006223422507

21. Lonjon G, Boutron I, Trinquart L, et al. Comparison of treatment effect estimates from prospective nonrandomized studies with propensity score analysis and randomized controlled trials of surgical procedures. Ann Surg. 2014;259(1):18-25. doi:10.1097/ SLA.0000000000000256

22. Schneeweiss S, Seeger JD, Landon J, Walker AM, Aprotinin during coronary-artery bypass grafting and risk of death. $N$ Engl J Med. 2008;358(8):771-783. doi:10.1056/NEJMoa0707571

23. Fergusson DA, Hébert PC, Mazer CD, et al. A comparison of aprotinin and lysine analogues in high-risk cardiac surgery. $N$ Engl J Med. 2008;358(22):2319-2331. doi:10.1056/NEJMoa0802395

24. Patorno E, Goldfine AB, Schneeweiss S, et al. Cardiovascular outcomes associated with canagliflozin versus other non-gliflozin antidiabetic drugs: Population Based Cohort Study. BMJ. 2018:360. Available from: https://pubmed.ncbi.nlm.nih.gov/29437648/.

25. Neal B, Perkovic V, Mahaffey KW, et al. Canagliflozin and cardiovascular and renal events in type 2 diabetes. $N$ Engl J Med. 2017;377 (7):644-657. doi:10.1056/NEJMoa1611925

26. Cohen AT, Goto S, Schreiber K, Torp-Pedersen C. Why do we need observational studies of everyday patients in the real-life setting? Eur Hear J. 2015;17(suppl D):D2-D8. doi:10.1093/eurheartj/suv035

27. Fong LCW, Ford TJ, da Costa BR, Jüni P, Berry C. Bias and loss to follow-up in cardiovascular randomized trials: a systematic review. $J$ Am Heart Assoc. 2020;9(14). doi: 10.1161/JAHA.119.015361

28. Balk EM, Bonis PAL, Moskowitz H, et al. Correlation of quality measures with estimates of treatment effect in meta-analyses of randomized controlled trials. $J$ Am Med Assoc. 2002;287 (22):2973-2982. doi:10.1001/jama.287.22.2973

29. Gadde KM, Allison DB, Ryan DH, et al. Effects of low-dose, controlled-release, phentermine plus topiramate combination on weight and associated comorbidities in overweight and obese adults (CONQUER): a randomised, placebo-controlled, Phase 3 trial. Lancet. 2011;377(9774):1341-1352. doi:10.1016/S0140-6736(11)60205-5

30. Cunningham JW, Wiviott SD. Modern obesity pharmacotherapy: weighing cardiovascular risk and benefit. Clin Cardiol. 2014;37 (11):693-699. doi:10.1002/clc.22304 
31. Garvey WT, Ryan DH, Look M, et al. Two-year sustained weight loss and metabolic benefits with controlled-release phentermine/topiramate in obese and overweight adults (SEQUEL): a Randomized, Placebo-Controlled, Phase 3 Extension Study. Am J Clin Nutr. 2012;95(2):297-308. doi:10.3945/ajcn.111.024927

32. European Medicines Agency. Refusal of the Marketing Authorisation for Qsiva (Phentermine/Topiramate). EMA; 2013.

33. Halpern B, Mancini MC. Safety assessment of combination therapies in the treatment of obesity: focus on naltrexone/bupropion extended release and phentermine-topiramate extended release. Expert Opin Drug Saf. 2017;16(1):27-39. doi:10.1080/14740338.2017.1247807

34. Nissen SE, Wolski KE, Prcela L, et al. Effect of naltrexone-bupropion on major adverse cardiovascular events in overweight and obese patients with cardiovascular risk factors: a randomized clinical trial. JAMA J Am Med Assoc. 2016;315 (10):990. doi:10.1001/jama.2016.1558

35. ClinicalTrials.gov. Naltrexone/bupropion cardiovascular outcomes study [Internet]; 2017. Available from: https://clinicaltrials.gov/ct2/show/ NCT02638129? term $=$ contrave + cardiovascular\&rank $=1$. Accessed June 29, 2021.

36. Krentz AJ, Hompesch M. Targeting hyperglycaemia with anti-obesity drugs: time for a paradigm shift? Drugs. 2013;73(15):1649-1651. doi:10.1007/s40265-013-0123-2

37. Røder ME. Major adverse cardiovascular event reduction with GLP-1 and SGLT2 agents: evidence and clinical potential. Ther Adv Chronic Dis. 2018;9(1):33-50. doi:10.1177/2040622317735283

38. Sandoval DA, D'Alessio DA. Physiology of proglucagon peptides: role of glucagon and GLP-1 in health and disease. Physiol Rev. 2015;95(2):513-548. doi:10.1152/physrev.00013.2014

39. Van Can J, Sloth B, Jensen CB, Flint A, Blaak EE, Saris WHM. Effects of the once-daily GLP-1 analog liraglutide on gastric emptying, glycemic parameters, appetite and energy metabolism in obese, non-diabetic adults. Int J Obes. 2014;38(6):784-793. doi:10.1038/ijo.2013.162

40. Scott LJ. Liraglutide: a review of its use in the management of obesity. Drugs. 2015;75(8):899-910. doi:10.1007/s40265-015-0408-8

41. Pi-Sunyer X, Astrup A, Fujioka K, et al. A randomized, controlled trial of $3.0 \mathrm{mg}$ of liraglutide in weight management. $N$ Engl J Med. 2015;373(1):11-22. doi:10.1056/NEJMoa1411892

42. Kelly AS, Auerbach P, Barrientos-Perez M, Gies I, Hale PM, Marcus C. A randomized, controlled trial of liraglutide for adolescents with obesity. $N$ Engl J Med. 2020;382(22):2117-2128. doi:10.1056/NEJMoa1916038

43. Novo Nordisk A/S. Novo Nordisk A/S: CHMP endorses EU label update of Saxenda ${ }^{\circledR}$ based on the LEADER trial 2017. 2017.

44. Davies MJ, Aronne LJ, Caterson ID, Thomsen AB, Jacobsen PB, Marso SP. Liraglutide and cardiovascular outcomes in adults with overweight or obesity: a post hoc analysis from SCALE randomized controlled trials. Diabetes Obes Metab. 2018;20(3):734-739. doi:10.1111/dom. 13125

45. O’Neil PM, Birkenfeld AL, McGowan B, et al. Efficacy and safety of semaglutide compared with liraglutide and placebo for weight loss in patients with obesity: a randomised, double-blind, placebo and active controlled, dose-ranging, Phase 2 trial. Lancet. 2018;392 (10148):637-649. doi:10.1016/S0140-6736(18)31773-2
46. Marso SP, Bain SC, Consoli A, et al. Semaglutide and cardiovascular outcomes in patients with type 2 diabetes. N Engl J Med. 2016.

47. Marso SP, Daniels GH, Brown-Frandsen K, et al. Liraglutide and cardiovascular outcomes in type 2 diabetes. N Engl J Med. 2016;375 (4): 311-322.:

48. Bain SC, Mosenzon O, Arechavaleta R, et al. Cardiovascular safety of oral semaglutide in patients with type 2 diabetes: rationale, design and patient baseline characteristics for the PIONEER 6 trial. Diabetes Obes Metab. 2019;21(3):499-508. doi:10.1111/dom.13553

49. Wilding JPH, Batterham RL, Calanna S, Davies M, Van Gaal LF, Lingvay I. Once-weekly semaglutide in adults with overweight or obesity. $N$ Engl J Med. 2021;384(11):989-1002.

50. Davies M, Færch L, Jeppesen OK, et al. Semaglutide $2.4 \mathrm{mg}$ once a week in adults with overweight or obesity, and type 2 diabetes (STEP 2): a randomised, double-blind, double-dummy, placebocontrolled, phase 3 trial. Lancet. 2021;397(10278):971-984. doi:10.1016/S0140-6736(21)00213-0

51. Wadden TA, Bailey TS, Billings LK, et al. Effect of subcutaneous semaglutide vs placebo as an adjunct to intensive behavioral therapy on body weight in adults with overweight or obesity: the STEP 3 randomized clinical trial. JAMA J Am Med Assoc. 2021;325 (14):1403-1413. doi:10.1001/jama.2021.1831

52. Rubino D, Abrahamsson N, Davies M, et al. Effect of continued weekly subcutaneous semaglutide vs placebo on weight loss maintenance in adults with overweight or obesity: the STEP 4 randomized clinical trial. JAMA J Am Med Assoc. 2021;325(14):1414-1425. doi:10.1001/jama.2021.3224

53. Ingelfinger JR, Rosen CJ. STEP 1 for effective weight control another first step? $N$ Engl J Med. 2021;384(11):1066-1067. doi:10.1056/NEJMe2101705

54. Ferrannini E, Solini A. SGLT2 inhibition in diabetes mellitus: rationale and clinical prospects. Nat Rev Endocrinol. 2012;8(8):495-502. doi:10.1038/nrendo.2011.243

55. Zinman B, Wanner C, Lachin JM, et al. Empagliflozin, cardiovascular outcomes, and mortality in type 2 diabetes. $N$ Engl $\mathrm{J}$ Med. 2015;373(22):2117-2128. doi:10.1056/NEJMoa1504720

56. Rådholm K, Figtree G, Perkovic V, et al. Canagliflozin and heart failure in type 2 diabetes mellitus. Circulation. 2018;138(5):458-468. doi:10.1161/CIRCULATIONAHA.118.034222

57. McMurray JJV, Solomon SD, Inzucchi SE, et al. Dapagliflozin in patients with heart failure and reduced ejection fraction. $N$ Engl J Med. 2019;381(21):1995-2008. doi:10.1056/NEJMoa1911303

58. Wang B, Zhong J, Lin H, et al. Blood pressure-lowering effects of GLP-1 receptor agonists exenatide and liraglutide: a meta-analysis of clinical trials. Diabetes Obes Metab. 2013;15(8):737-749. doi:10.1111/dom. 12085

59. Cannon CP, Pratley R, Dagogo-Jack S, et al. Cardiovascular outcomes with ertugliflozin in type 2 diabetes. $N$ Engl $J$ Med. 2020;383(15):1425-1435. doi:10.1056/NEJMoa2004967

\section{Publish your work in this journal}

Diabetes, Metabolic Syndrome and Obesity: Targets and Therapy is an international, peer-reviewed open-access journal committed to the rapid publication of the latest laboratory and clinical findings in the fields of diabetes, metabolic syndrome and obesity research. Original research, review, case reports, hypothesis formation, expert opinion and commentaries are all considered for publication. The manuscript management system is completely online and includes a very quick and fair peer-review system, which is all easy to use. Visit http://www.dovepress.com/testimonials.php to read real quotes from published authors. 